\title{
Factors related to compliance with adjuvant chemotherapy in patients with gastric cancer: A retrospective single-center study
}

\author{
Geon Yi Jin', Ki Bum Park², Kyo Young Song' \\ 'Department of Surgery, Seoul St. Mary's Hospital, College of Medicine, The Catholic University of Korea, Seoul; \\ ${ }^{2}$ Department of Surgery, St. Vincent's Hospital, College of Medicine, The Catholic University of Korea, Seoul, Korea
}

Purpose: Adjuvant chemotherapy (AC) improves survival outcomes in patients with advanced gastric cancer (GC) after curative surgery; however, some patients do not receive or complete chemotherapy. This study aimed to identify factors related to patient compliance with chemotherapy after curative surgery for advanced GC.

Methods: The data of patients who underwent curative gastrectomy for pathologic stage II-III GC between 2012 and 2016 were reviewed. Patients were divided into an AC completion group (group C), AC incompletion group (group I), and surgery-only group (group S). The AC regimen was either tegafur/gimeracil/oteracil (S-1) or capecitabine plus oxaliplatin (XELOX).

Results: The study enrolled 417 patients; group $C$ had 222 patients, group I had 110, and group $S$ had 85. The most common reason for not initiating AC was poor general condition (36.5\%), while chemotherapy-related complications was the common reason for AC incompletion (43.6\%). In multivariate analysis, age over 65 years, Eastern Cooperative Oncology Group performance status $\geq 1$, Charlson comorbidity index $\geq 1$, and the presence of postoperative complications were independent risk factors for not initiating AC (odds ratio: 4.32, 2.62, 1.84, and 2.17, respectively). Age over 65 years, longer postoperative stay, and XELOX regimen were significant risk factors for incompletion of AC (odds ratio: $2.68,1.72$, and 2.23 , respectively).

Conclusion: Old age, poor performance status, comorbidities, and postoperative complications, longer postoperative hospital stay, and XELOX regimen were associated with poor compliance with $\mathrm{AC}$ in $\mathrm{GC}$ patients. Clinicians can improve compliance with $\mathrm{AC}$ by managing postoperative complications and selecting the most appropriate treatment regimen.

Keywords: Gastric cancer, Gastrectomy, Adjuvant chemotherapy, Patient compliance

\section{INTRODUCTION}

Radical gastrectomy is the standard treatment for resectable gastric cancer (GC). South Korean and Japanese guidelines recommend

Received: Apr 6, 2021 Revised: Jun 23, 2021 Accepted: Jun 26, 2021 Correspondence to: : Kyo Young Song

Department of Surgery, Seoul St. Mary's Hospital, College of Medicine, The Catholic University of Korea, 222 Banpo-daero, Seocho-gu, Seoul 06591, Korea

Tel: +82-2-2258-6238; Fax: +82-2-595-2822

E-mail: skygs@catholic.ac.kr; skys9615@gmail.com

ORCID: Geon Yi Jin (https://orcid.org/0000-0002-1705-5513), Ki Bum Park (https:// orcid.org/0000-0001-6035-6584), Kyo Young Song (https://orcid.org/0000-00025840-1638)

\section{Copyright (C) 2021 Korean Society of Surgical Oncology}

This is an Open Access article distributed under the terms of the Creative Commons Attribution Non-Commercial License (http://creativecommons.org/licenses/by-nc/4.0) which permits unrestricted non-commercial use, distribution, and reproduction in any medium, provided the original work is properly cited. adjuvant chemotherapy (AC) after D2 lymph node dissection for patients with pathologic stage II-III to improve survival outcomes [1,2]. In 2007, a randomized clinical trial of tegafur/gimeracil/oteracil (S-1) therapy, the ACTS-GC trial, demonstrated a survival benefit of adjuvant oral fluoropyrimidine therapy compared with surgery alone [3]. Similarly, the CLASSIC trial found that AC with capecitabine plus oxaliplatin (XELOX) after gastrectomy with D2 lymph node dissection should be considered for stage II-III patients [4].

To maximize the benefits of chemotherapy, appropriate treatment schedules and good patient compliance are important. Most studies on the efficacy of XELOX and S-1 were based on eight cycles of AC. Recent studies have shown that starting AC within 8 weeks of surgery, and continuing treatment for more than 6 months, improves survival rates $[5,6]$. Other studies reported that the completion rate for eight cycles of S- 1 or XELOX is approximately $65 \%-75 \%[7,8]$. Therefore, $25 \%-35 \%$ of patients do not 
complete or initiate AC for various reasons, including poor physical condition before or after gastrectomy.

Given the current paucity of data, this study aimed to identify factors associated with compliance with AC after gastrectomy for GC.

\section{METHODS}

\section{Patients}

This single-center cohort study included 440 patients diagnosed with pathologic stage II-III GC, who underwent curative radical gastrectomy at Seoul St. Mary's Hospital from 2012 to 2016. The exclusion criteria were as follows: previously received neoadjuvant chemotherapy, diagnosed with cancer in another organ within 5 years prior to gastrectomy, and missing data due to death or loss to follow-up. Ultimately, 417 patients were evaluated retrospectively.

\section{Study protocol}

Eligible patients were divided into three groups: 222 patients received eight cycles of AC (completion group, group C), while in 110 patients AC was initiated but they ultimately received less than eight cycles of chemotherapy (incompletion group, group I). Finally, 85 patients only underwent gastrectomy without AC (surgery-only group, group S). Baseline characteristics (at the time of gastrectomy) and operative outcomes were extracted from the electric medical records. Comorbidities were quantified using the Charlson comorbidity index (CCI) [9]. The physical status of patients was categorized using the Eastern Cooperative Oncology Group performance status (ECOG-PS). The severity of postoperative complications was classified according to the Clavien-Dindo classification (CDC) [10]. CDC grade $\geq 3$ was taken to indicate severe complications. Depth of invasion and lymph node metastasis were categorized according to the 8th American Joint Committee on Cancer TNM classification. The reasons for discontinuing chemotherapy and not initiating chemotherapy were investigated in groups I and S, respectively. After the AC treatment, patients were followed up regularly according to the standard protocols of our institution. Patients visited the outpatient clinic every 3 months for the first 2 years, and every 6 months thereafter. The median follow-up period was 42 months. This study was approved by the Ethics Committee and Institutional Review Board of Seoul St. Mary's Hospital (approval No. KC20RASI0937). The informed consent was waived.

\section{Chemotherapy protocols}

AC was planned for patients diagnosed with stage II-III GC after curative surgery. According to the policy of our institute, S-1 thera- py was mainly used for stage II patients, and XELOX therapy for stage III patients. The treatment generally began about 4 weeks after surgery. The chemotherapy protocols of our institution are as follows. The S-1 regimen consists of eight 4-week cycles of 80-120 mg oral S-1 per square meter of body surface area per day, followed by a "rest period" of 2 weeks. The XELOX regimen consists of eight 3-week cycles of oral capecitabine $\left(1,000 \mathrm{mg} / \mathrm{m}^{2}\right.$ twice daily on days 1-14 of each cycle) plus intravenous oxaliplatin (130 mg/ $\mathrm{m}^{2}$ on day 1 of each cycle), followed by a rest period of 1 week. Side effects were assessed based on blood tests performed at the time of initiation of each cycle and 1 week thereafter. Cancer progression was checked using computed tomography and tumor marker tests performed after every three cycles of chemotherapy.

\section{Statistical analysis}

The data were analyzed using SPSS for Windows software version 24.0 (IBM Corp., Armonk, NY, USA). Continuous variables were analyzed using Student t-test or one-way analysis of variance. Categorical variables were compared with the chi-square test or Fisher exact test. The Bonferroni post hoc test was also applied. Overall survival (OS) was calculated using the Kaplan-Meier method, and compared among groups using the log-rank test. Factors that differed significantly among groups were subjected to multivariate analysis, with logistic regression used to determine risk factors for chemotherapy compliance and calculate the odds ratios (ORs). $\mathrm{P}$-value $<0.05$ was considered statistically significant, and $\mathrm{P}$ $<0.017$ was considered significant in the post hoc analysis.

\section{RESULTS}

The median follow-up duration was 42 months (range, 1-92 months). For groups $\mathrm{C}, \mathrm{I}$ and $\mathrm{S}$, the 5 -year OS rate was $85.5 \%$, $80.6 \%$, and $64.6 \%$, respectively $(\mathrm{P}<0.001)$ (Supplementary Fig. S1). The baseline characteristics and operative details of the three groups are shown in Table 1. The mean age of groups C, I, and S was $56.6,60.5$, and 72.1 years, respectively; group $S$ patients were significantly older than those on the other groups $(\mathrm{P}<0.001)$. The ECOG-PS and CCI were significantly different among the three groups, and group $S$ had the highest proportions of patients with an ECOG-PS or CCI $\geq 1$ (64.7\% and 51.8\%, respectively). The mean postoperative length of stay (PLOS) was significantly longer in group $S$ than groups $C$ and I (13.1, 8.7 and 9.7 days, respectively, $\mathrm{P}<0.001)$. The proportion of patients receiving $\mathrm{S}-1$ was significantly higher in group $\mathrm{C}$ than group I (64.0\% and 51.8\%, respectively, $\mathrm{P}=0.034)$. The most common reasons for not receiving chemotherapy were poor general condition (36.5\%) and patient refusal (29.4\%), while chemotherapy-related complications was the ma- 
Table 1. Comparison of baseline characteristics between three groups

\begin{tabular}{|c|c|c|c|c|}
\hline Variable & $\begin{array}{l}\text { Completion group } \\
\qquad(n=222)\end{array}$ & $\begin{array}{l}\text { Incompletion group } \\
\qquad(n=110)\end{array}$ & $\begin{array}{l}\text { Surgery only group } \\
\qquad(n=85)\end{array}$ & $\mathrm{P}$-value \\
\hline Age (yr) & $56.6 \pm 11.5$ & $60.5 \pm 12.6$ & $72.1 \pm 11.2$ & $0.001^{\text {a) }}$ \\
\hline Sex & & & & 0.719 \\
\hline Male & $148(66.7)$ & $77(70.0)$ & $55(64.7)$ & \\
\hline Female & 74 (33.3) & $33(30.0)$ & $30(35.3)$ & \\
\hline $\mathrm{BMI}\left(\mathrm{kg} / \mathrm{m}^{2}\right)$ & $23.4 \pm 3.1$ & $23.1 \pm 3.6$ & $23.3 \pm 3.4$ & 0.729 \\
\hline ECOG-PS & & & & $0.001^{\mathrm{a})}$ \\
\hline 0 & $180(81.1)$ & 78 (70.9) & $30(35.3)$ & \\
\hline$\geq 1$ & $42(18.9)$ & $32(29.1)$ & $55(64.7)$ & \\
\hline $\mathrm{CCl}$ & & & & $0.001^{\mathrm{a}, \mathrm{b})}$ \\
\hline 0 & $170(76.6)$ & 75 (68.2) & $41(48.2)$ & \\
\hline$\geq 1$ & $52(23.4)$ & $35(31.8)$ & 44 (51.8) & \\
\hline Approach & & & & 0.543 \\
\hline Laparoscopic & $159(71.6)$ & $85(77.3)$ & $63(74.1)$ & \\
\hline Open & $63(28.4)$ & $25(22.7)$ & $22(25.9)$ & \\
\hline Extent of resection & & & & 0.400 \\
\hline Subtotal & 73 (32.9) & 43 (39.1) & $26(30.6)$ & \\
\hline Total & $149(61.7)$ & $67(60.9)$ & $59(69.4)$ & \\
\hline LN dissection & & & & 0.318 \\
\hline$\leq \mathrm{D} 1+$ & $32(14.4)$ & $16(14.5)$ & $18(21.2)$ & \\
\hline$\geq \mathrm{D} 2$ & $190(85.6)$ & $94(85.5)$ & 67 (78.8) & \\
\hline Reconstruction & & & & 0.078 \\
\hline$B-1$ & $18(8.1)$ & $2(1.8)$ & $4(4.7)$ & \\
\hline$B-\|$ & $125(56.3)$ & $62(56.4)$ & $56(65.9)$ & \\
\hline$R-Y$ & 79 (35.6) & $46(41.8)$ & 25 (29.4) & \\
\hline Pathologic stage & & & & 0.094 \\
\hline$\|$ & $108(48.6)$ & 43 (39.1) & $46(54.1)$ & \\
\hline III & $114(51.4)$ & $67(60.9)$ & $39(45.9)$ & \\
\hline Postoperative complications & $62(27.9)$ & $34(30.9)$ & $40(47.1)$ & $0.006^{a), c]}$ \\
\hline $\mathrm{CDC} \geq 3$ & $19(30.6)$ & $11(32.4)$ & $14(35.0)$ & 0.900 \\
\hline Postoperative hospital stay (day) & $8.7 \pm 3.2$ & $9.7 \pm 4.0$ & $13.1 \pm 11.8$ & $0.001^{\mathrm{a}), \mathrm{d})}$ \\
\hline Interval from operation to chemotherapy (day) & $36.4 \pm 10.4$ & $37.8 \pm 10.6$ & & 0.289 \\
\hline Chemotherapy regimen & & & & $0.034^{\mathrm{a})}$ \\
\hline S-1 & $142(64.0)$ & $57(51.8)$ & & \\
\hline XELOX & $80(36.0)$ & $53(48.2)$ & & \\
\hline
\end{tabular}

Values are presented as mean \pm standard deviation or number $(\%)$.

BMI, body mass index; ECOG-PS, Eastern Cooperative Oncology Group performance status; CCI Charlson comorbidity index; LN, lymph node; B-I, Billroth I; B-II, Billroth II; R-Y, Roux-en-Y; CDC, Clavien-Dindo classification; S-1, tegafur/gimeracil/oteracil; XELOX, capecitabine plus oxaliplatin.

a) Statistically significant, $\mathrm{P}<0.05$. ${ }^{\text {b) }}$ Completion group vs. incompletion group $=0.102$; incompletion group vs. surgery only group $=0.001$; completion group vs. surgery only group $=0.003$. Completion group vs. incompletion group $=0.608$; incompletion group vs. surgery only group $=0.026$; completion group vs. surgery only group $=0.002$. ${ }^{\text {d) }}$ Completion group vs. incompletion group $=0.436$; incompletion group vs. surgery only group $=0.001$; completion group vs. surgery only group $=0.003$.

jor reason for incompletion thereof (43.6\%) (Table 2).

An age over 65 years, PLOS over 7 days, and use of XELOX were significant risk factors for incompletion of AC (OR: 2.68, 1.72, and 2.23 , respectively) (Table 3 ). An age over 65 years, ECOG-PS $\geq 1$, $\mathrm{CCI} \geq 1$, and presence of postoperative complications were significant risk factors for not initiating AC (OR: 4.32, 2.62, 1.84, and
2.17 , respectively) (Table 4).

\section{DISCUSSION}

The benefits of AC for advanced GC have been proven in largescale, randomized controlled trials. However, some patients do not 
Table 2. Reasons for not completing adjuvant chemotherapy for group I and group $S$

\begin{tabular}{lc}
\hline Reason & No. of patients (\%) \\
\hline Reasons for incompletion (group I, $n=110)$ & \\
Chemotherapy-related complications & $48(43.6)$ \\
Gastro-intestinal symptoms & $23(47.9)$ \\
Poor oral intake & $14(29.2)$ \\
Skin eruption & $8(16.7)$ \\
Neuropathy & $3(6.3)$ \\
Patient's refusal & $20(18.2)$ \\
Disease progression & $12(10.9)$ \\
Surgery-related complication & $5(4.5)$ \\
Others & $25(22.7)$ \\
Reasons for no initiation (group S, $n=85)$ & \\
Poor condition & $31(36.5)$ \\
Patient's refusal & $25(29.4)$ \\
Surgery-related complication & $10(11.8)$ \\
Others & $19(22.4)$ \\
\hline
\end{tabular}

receive chemotherapy for various reasons, resulting in poor survival outcomes. This study investigated factors associated with compliance with AC. In multivariate analysis, old age, comorbidities, and postoperative complications were risk factors for not initiating AC. Old age, long PLOS, and XELOX regimen were risk factors for discontinuing AC. Clinicians should pay close attention to patients with such risk factors and try to prevent those that are controllable, such as postoperative complications and PLOS.

In this study, old age and comorbidities were risk factors for not initiating chemotherapy (Supplementary Tables S1, S2). One of the most common sequelae after GC surgery is dietary problems, which can lead to weight loss and sarcopenia [11]. A previous study reported that postoperative weight loss can affect compliance with AC in GC patients [12]. Older patients with comorbidities are more likely to experience postoperative dietary problems or severe weight loss, which may reduce compliance with chemotherapy. A previous study reported that old age ( $>60$ years), low body mass index $\left(<23 \mathrm{~kg} / \mathrm{m}^{2}\right)$, and poor physical status were associated with lower compliance [13]. Other studies on the S-1 regimen for GC patients reported than an age over 65 years was a significant factor for poor compliance with AC $[14,15]$.

Prolonged PLOS was a risk factor for incompletion of AC in this study. Gastrectomy patients in our institution are discharged on postoperative day 7 following application of the clinical protocol. Therefore, a PLOS over 7 days indicates problems such as operative complications or delayed recovery. Although the proportion of patients with a CDC grade $\geq 3$ did not differ significantly among the three groups, that was not the case for CDC grade $\geq 1$ patients. This suggests that minor complications, such as gastric stasis and
Table 3. Multivariable analysis of factors for incompletion of adjuvant chemotherapy

\begin{tabular}{lccc}
\hline Variable & OR & $95 \%$ Cl & P-value \\
\hline Age $>65$ yr & 2.68 & $1.57-4.58$ & $<0.001^{\text {a) }}$ \\
ECOG-PS 1 or more & 1.38 & $0.71-2.60$ & 0.279 \\
CCl 1 or more & 1.44 & $0.84-2.48$ & 0.147 \\
Postoperative complication & 1.07 & $0.47-2.45$ & 0.393 \\
Postoperative admission day $>7$ & 1.72 & $1.04-2.85$ & $0.034^{\text {a) }}$ \\
XELOX regimen & 2.23 & $1.33-3.73$ & $0.002^{\text {a) }}$ \\
\hline
\end{tabular}

$\mathrm{OR}$, odds ratio; $\mathrm{Cl}$, confidence interval; ECOG-PS, Eastern Cooperative Oncology Group performance status; CCl, Charlson comorbidity index; XELOX, capecitabine plus oxaliplatin.

a) Statistically significant, $\mathrm{P}<0.05$.

Table 4. Multivariable analysis of factors for no initiation of adjuvant chemotherapy

\begin{tabular}{lccc}
\hline Variable & OR & $95 \%$ Cl & P-value \\
\hline Age $>65$ yr & 4.32 & $2.23-8.34$ & $<0.001^{\text {a) }}$ \\
ECOG-PS 1 or more & 2.62 & $1.41-4.88$ & $0.002^{\mathrm{a})}$ \\
CCl 1 or more & 1.84 & $1.06-3.21$ & $0.030^{\mathrm{a})}$ \\
Postoperative complication & 2.17 & $1.24-3.78$ & $0.006^{\mathrm{a}}$ \\
Postoperative admission day $>7$ & 1.53 & $0.80-2.93$ & 0.197 \\
\hline
\end{tabular}

$\mathrm{OR}$, odds ratio; $\mathrm{Cl}$, confidence interval; ECOG-PS, Eastern Cooperative Oncology Group performance status; $\mathrm{CCl}$, Charlson comorbidity index.

a) Statistically significant, $\mathrm{P}<0.05$.

temporary ileus, can also affect postoperative recovery and the patient's condition. As postoperative complication was a risk factor for incompletion of $\mathrm{AC}$, clinicians should pay close attention to minor complications and complaints after surgery to enhance compliance.

The XELOX was applied in stage III GC patients and S- 1 was applied in stage II GC patients at our institution, respectively. A previous study reported that neither of these two regimens was significantly superior to the other [16]. However, other studies have reported that the XELOX regimen may be more effective in stage IIIB and IIIC GC patients, supporting the approach of our institution [7]. In this study, patients on the XELOX regimen were more likely to fail to complete chemotherapy than those on the S-1 regimen. The rate of dose reduction was higher in the XELOX than S-1 group (15\% vs. 5.5\%, P=0.004) (Supplementary Table S3), suggesting that the XELOX regimen was generally more difficult for patients to maintain. In the CLASSIC trial, grade 3-4 chemotherapy-related adverse events were reported in $56 \%$ of the XELOX group, compared to $22.8 \%$ of the $\mathrm{S}$ - 1 group in the ACTS-GC trial. Jang et al. [14] reported a more than two-fold higher rate of grade 3-4 chemotherapy-related adverse events in their XELOX group than the S-1 group (47\% vs. 21\%) [17]. Finally, oncologic pa- 
tients tend to prefer oral over intravenous drugs for reasons of convenience, perceived efficacy, and past experience [18]. Therefore, clinicians need to pay more attention to patients on the XELOX regimen.

Thirty-three patients stopped chemotherapy in the first cycle, which was the highest drop-out rate in group I (30\%) (Supplementary Fig. S2). Almost two-thirds of patients failed to complete more than half of the entire chemotherapy cycles $(70 \%, n=77)$. This suggest that patients may be more vulnerable to treatment in the early period. Therefore, it is necessary to focus more on patients with risk factors at the beginning of treatment.

This study had some limitations. First, it used as a retrospective, single center design, which could give rise to certain biases. Some factors found to be significant in other studies, including postoperative weight change, serum creatinine, and sarcopenia, were not analyzed in our study [19]. Second, the results of this study may not generalize to Western countries. While AC is frequently used in Asian countries, perioperative chemotherapy and adjuvant chemoradiation are used more often in Western countries [20,21]. Further research is also needed on the associations of health insurance and socioeconomic status with treatment outcomes.

In conclusion, an age over 65 years, prolonged PLOS, comorbidities, postoperative complications and XELOX treatment were associated with poorer compliance with AC in advanced GC patients. Compliance can be improved by treating postoperative complications appropriately, reducing the postoperative hospital stay, and selecting the appropriate treatment regimen according to the patient's life expectancy and systemic conditions.

\section{CONFLICT OF INTEREST}

No potential conflict of interest relevant to this article was reported.

\section{SUPPLEMENTARY MATERIALS}

Supplementary materials are available at the Korean Journal of Clinical Oncology website (http://www.kjco.org/).

\section{REFERENCES}

1. Guideline Committee of the Korean Gastric Cancer Association (KGCA), Development Working Group \& Review Panel. Korean practice guideline for gastric cancer 2018: an evidence-based, multi-disciplinary approach. J Gastric Cancer 2019;19:1-48.

2. Japanese Gastric Cancer Association. Japanese gastric cancer treatment guidelines 2014 (ver. 4). Gastric Cancer 2017;20:1-19.

3. Sakuramoto S, Sasako M, Yamaguchi T, Kinoshita T, Fujii M,
Nashimoto A, et al. Adjuvant chemotherapy for gastric cancer with S-1, an oral fluoropyrimidine. N Engl J Med 2007;357:1810-20.

4. Bang YJ, Kim YW, Yang HK, Chung HC, Park YK, Lee KH, et al. Adjuvant capecitabine and oxaliplatin for gastric cancer after D2 gastrectomy (CLASSIC): a phase 3 open-label, randomised controlled trial. Lancet 2012;379:315-21.

5. Ahn GT, Baek SK, Han JJ, Kim HJ, Jeong SJ, Maeng CH. Optimal time interval from surgery to adjuvant chemotherapy in gastric cancer. Oncol Lett 2020;20:32.

6. Huang SM, Chen YC, Chen WY, Yang LY, Tsan DL, Tsang NM, et al. Optimal timing for postsurgical adjuvant therapy in patients with gastric cancer: a propensity score matching study. J Cancer 2019;10:332-40.

7. Kim IH, Park SS, Lee CM, Kim MC, Kwon IK, Min JS, et al. Efficacy of adjuvant S-1 versus XELOX chemotherapy for patients with gastric cancer after D2 lymph node dissection: a retrospective, multi-center observational study. Ann Surg Oncol 2018;25:117683.

8. Lee CM, Yoo MW, Son YG, Oh SJ, Kim JH, Kim HI, et al. Longterm efficacy of S-1 monotherapy or capecitabine plus oxaliplatin as adjuvant chemotherapy for patients with stage II or III gastric cancer after curative gastrectomy: a propensity score-matched multicenter cohort study. J Gastric Cancer 2020;20:152-64.

9. Sundararajan V, Henderson T, Perry C, Muggivan A, Quan H, Ghali WA. New ICD-10 version of the Charlson comorbidity index predicted in-hospital mortality. J Clin Epidemiol 2004;57:1288-94.

10. Clavien PA, Barkun J, de Oliveira ML, Vauthey JN, Dindo D, Schulick RD, et al. The Clavien-Dindo classification of surgical complications: five-year experience. Ann Surg 2009;250:187-96.

11. Climent M, Munarriz M, Blazeby JM, Dorcaratto D, Ramon JM, Carrera MJ, et al. Weight loss and quality of life in patients surviving 2 years after gastric cancer resection. Eur J Surg Oncol 2017; 43:1337-43

12. Aoyama T, Kawabe T, Fujikawa H, Hayashi T, Yamada T, Tsuchida $\mathrm{K}$, et al. Loss of lean body mass as an independent risk factor for continuation of S-1 adjuvant chemotherapy for gastric cancer. Ann Surg Oncol 2015;22:2560-6.

13. Kim DW, Kwon OK, Yoo MW, Ryu SW, Oh SJ, Hur H, et al. Actual compliance to adjuvant chemotherapy in gastric cancer. Ann Surg Treat Res 2019;96:185-90.

14. Jang SH, Jung YJ, Kim MG, Kwon SJ. The prognostic significance of compliance with postoperative adjuvant chemotherapy in patients with stage III gastric cancer: an observational study. J Gastric Cancer 2018;18:48-57.

15. Yamashita K, Kurokawa Y, Yamamoto K, Hirota M, Kawabata R, Mikami J, et al. Risk factors for poor compliance with adjuvant S-1 chemotherapy for gastric cancer: a multicenter retrospective study. 
Ann Surg Oncol 2017;24:2639-45.

16. Nakamura Y, Yamanaka T, Chin K, Cho H, Katai H, Terashima M, et al. Survival outcomes of two phase 2 studies of adjuvant chemotherapy with S-1 plus oxaliplatin or capecitabine plus oxaliplatin for patients with gastric cancer after D2 gastrectomy. Ann Surg Oncol 2019;26:465-72.

17. Cho JH, Lim JY, Cho JY. Comparison of capecitabine and oxaliplatin with S-1 as adjuvant chemotherapy in stage III gastric cancer after D2 gastrectomy. PLoS One 2017;12:e0186362.

18. Eek D, Krohe M, Mazar I, Horsfield A, Pompilus F, Friebe R, et al. Patient-reported preferences for oral versus intravenous administration for the treatment of cancer: a review of the literature. Patient
Prefer Adherence 2016;10:1609-21.

19. Aoyama T, Yoshikawa T, Hayashi T, Kuwabara H, Mikayama Y, Ogata T, et al. Risk factors for 6-month continuation of S-1 adjuvant chemotherapy for gastric cancer. Gastric Cancer 2013;16:1339.

20. Bickenbach K, Strong VE. Comparisons of gastric cancer treatments: east vs. west. J Gastric Cancer 2012;12:55-62.

21. Mueller JL, Kim DH, Stapleton S, Cauley CE, Chang DC, Park $\mathrm{CH}$, et al. Nature versus nurture: the impact of nativity and site of treatment on survival for gastric cancer. Gastric Cancer 2019;22: 446-55. 